\title{
Six unified results for reducibility of the Srivastava's triple hypergeometric series $H_{A}$
}

Junesang Choi ${ }^{a, *}$, Arjun K. Rathie ${ }^{b}$

${ }^{a}$ Department of Mathematics, Dongguk University, Gyeongju 38066, Republic of Korea.

${ }^{b}$ Department of Mathematics, School of Physical Sciences, Central University of Kerala, Kasaragod-671316, Kerala, India.

Communicated by Y. J. Cho

\begin{abstract}
We aim to provide six unified results for reducibility of the Srivastava's triple hypergeometric series $\mathrm{H}_{\mathrm{A}}$. The results are obtained with the help of generalizations of classical summation theorems due to Kummer, Gauss second and Bailey for the series ${ }_{2} F_{1}$ which have recently been published. Our main findings are also shown to be specialized to yield several known results. (C)2017 All rights reserved.
\end{abstract}

Keywords: Gamma function, hypergeometric function, generalized hypergeometric function, summation theorems, Appell's function $\mathrm{F}_{1}$, triple hypergeometric series $\mathrm{H}_{\mathrm{A}}$.

2010 MSC: 33C60, 33C65, 33C05, 33C70.

\section{Introduction and preliminaries}

We begin by recalling the generalized hypergeometric series ${ }_{p} F_{q}$ defined by $($ see $[1,3,11]$ and $[17$, pp. 71-75]):

$$
\begin{aligned}
{ }_{p} F_{q}\left[\begin{array}{l}
\alpha_{1}, \cdots, \alpha_{p} ; z \\
\beta_{1}, \cdots, \beta_{q} ;
\end{array}\right] & =\sum_{n=0}^{\infty} \frac{\left(\alpha_{1}\right)_{n} \cdots\left(\alpha_{p}\right)_{n}}{\left(\beta_{1}\right)_{n} \cdots\left(\beta_{q}\right)_{n}} \frac{z^{n}}{n !} \\
& ={ }_{p} F_{q}\left(\alpha_{1}, \cdots, \alpha_{p} ; \beta_{1}, \cdots, \beta_{q} ; z\right),
\end{aligned}
$$

where $(\lambda)_{n}$ is the Pochhammer symbol defined (for $\lambda \in \mathbb{C}$ ) by (see [17, p. 2 and p. 5]):

$$
\begin{aligned}
(\lambda)_{n}: & = \begin{cases}1, & (n=0), \\
\lambda(\lambda+1) \cdots(\lambda+n-1), & (n \in \mathbb{N}),\end{cases} \\
& =\frac{\Gamma(\lambda+n)}{\Gamma(\lambda)}, \quad\left(\lambda \in \mathbb{C} \backslash \mathbb{Z}_{0}^{-}\right),
\end{aligned}
$$

\footnotetext{
*Corresponding author

Email addresses: junesang@mail.dongguk.ac.kr (Junesang Choi), akrathie@cukerala.ac.in (Arjun K. Rathie)
} doi:10.22436/jnsa.010.06.18 
and $\Gamma(\lambda)$ is the familiar Gamma function. Here and in the following, let $\mathbb{R}, \mathbb{C}, \mathbb{Z}$ and $\mathbb{N}$ be the sets of real numbers, complex numbers, integers and positive integers, respectively, and

$$
\mathbb{N}_{0}:=\mathbb{N} \cup\{0\} \quad \text { and } \quad \mathbb{Z}_{0}^{-}:=\mathbb{Z} \backslash \mathbb{N} \text {. }
$$

It is well-known and noted that whenever the generalized hypergeometric function with certain specified parameters and argument can be summed to be expressed in terms of the Gamma functions, the results play important roles in applications. For example, the classical summation theorems of Gauss, Kummer, Gauss second and Bailey for ${ }_{2} F_{1}$, and some other summations for ${ }_{3} F_{2}$, and ${ }_{4} F_{3}$ have been used in diverse ways (see, e.g., [11] and [17, p. 350]).

Here we recall the following classical summation theorems for the series ${ }_{2} \mathrm{~F}_{1}$ :

- Kummer's theorem (see, e.g., [11, p. 68]):

$$
\begin{gathered}
{ }_{2} \mathrm{~F}_{1}\left[\begin{array}{r}
\mathrm{a}, \mathrm{b} ; \\
1+\mathrm{a}-\mathrm{b} ;
\end{array}\right]=\frac{\Gamma\left(1+\frac{1}{2} \mathrm{a}\right) \Gamma(1+\mathrm{a}-\mathrm{b})}{\Gamma(1+\mathrm{a}) \Gamma\left(1+\frac{1}{2} \mathrm{a}-\mathrm{b}\right)}, \\
\left(\mathfrak{R}(\mathrm{b})<1 ; 1+\mathrm{a}-\mathrm{b} \in \mathbb{C} \backslash \mathbb{Z}_{0}^{-}\right) .
\end{gathered}
$$

- Gauss's second theorem (see, e.g., [15, p. 243]):

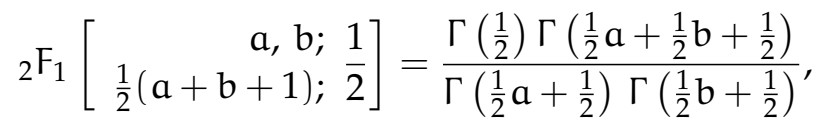

$$
\begin{aligned}
& \left(\frac{1}{2}(a+b+1) \in \mathbb{C} \backslash \mathbb{Z}_{0}^{-}\right) \text {. }
\end{aligned}
$$

- Bailey's theorem (see, e.g., [15, p. 243]):

$$
{ }_{2} F_{1}\left[\begin{array}{r}
a, 1-a ; 1 \\
c ; \frac{1}{2}
\end{array}\right]=\frac{\Gamma\left(\frac{1}{2} c\right) \Gamma\left(\frac{1}{2} c+\frac{1}{2}\right)}{\Gamma\left(\frac{1}{2} c+\frac{1}{2} a\right) \Gamma\left(\frac{1}{2} c-\frac{1}{2} a+\frac{1}{2}\right)}, \quad\left(c \in \mathbb{C} \backslash \mathbb{Z}_{0}^{-}\right) .
$$

For applications of these theorems (1.1), (1.2) and (1.3), one may refer to Bailey [2].

Recently a good deal of progress has been done in generalizing the above classical theorems. During 1992-96, in a series of three papers [7-9], Lavoie et al. have presented generalizations of the above-recalled classical summation theorems (1.1), (1.2) and (1.3), and Watson's, Dixon's and Whipple's summation theorems for ${ }_{3} \mathrm{~F}_{2}$, a large number of whose limiting and special cases were also considered.

In fact, Lavoie et al. have obtained explicit expressions (in terms of Gamma functions) of the following ${ }_{2} \mathrm{~F}_{1}$ :

$$
\begin{aligned}
& { }_{2} F_{1}\left[\begin{array}{r}
a, b ; \\
1+a-b+i ;
\end{array}-1\right], \\
& { }_{2} F_{1}\left[\begin{array}{r}
a, b ; \\
\frac{1}{2}(a+b+i+1) ;
\end{array}\right],
\end{aligned}
$$

and

$$
{ }_{2} F_{1}\left[\begin{array}{rr}
a, 1-a+i ; & 1 \\
c ; & 2
\end{array}\right]
$$

for $i=0, \pm 1, \cdots, \pm 5$.

Rakha and Rathie [12] further discovered the most general cases of the results (1.4), (1.5), (1.6) for any $i \in \mathbb{Z}$ which are recalled as follows: 
- Generalizations of Kummer's theorem:

$$
\begin{aligned}
{ }_{2} F_{1}\left[\begin{array}{r}
a, b ; \\
1+a-b+i ;
\end{array}=\right. & \frac{2^{-a} \Gamma\left(\frac{1}{2}\right) \Gamma(b-i) \Gamma(1+a-b+i)}{\Gamma(b) \Gamma\left(\frac{1}{2} a-b+\frac{1}{2} i+\frac{1}{2}\right) \Gamma\left(\frac{1}{2} a-b+\frac{1}{2} i+1\right)} \\
& \times \sum_{r=0}^{i}(-1)^{r}\left(\begin{array}{c}
i \\
r
\end{array}\right) \frac{\Gamma\left(\frac{1}{2} a-b+\frac{1}{2} i+\frac{1}{2} r+\frac{1}{2}\right)}{\Gamma\left(\frac{1}{2} a-\frac{1}{2} i+\frac{1}{2} r+\frac{1}{2}\right)}, \quad\left(i \in \mathbb{N}_{0}\right),
\end{aligned}
$$

and

$$
\begin{aligned}
{ }_{2} F_{1}\left[\begin{array}{r}
a, b ; \\
1+a-b-i ;
\end{array}=\right. & \frac{2^{-a} \Gamma\left(\frac{1}{2}\right) \Gamma(1+a-b-i)}{\Gamma\left(\frac{1}{2} a-b-\frac{1}{2} \mathfrak{i}+\frac{1}{2}\right) \Gamma\left(\frac{1}{2} a-b-\frac{1}{2} \mathfrak{i}+1\right)} \\
& \times \sum_{r=0}^{i}\left(\begin{array}{l}
i \\
r
\end{array}\right) \frac{\Gamma\left(\frac{1}{2} a-b-\frac{1}{2} \mathfrak{i}+\frac{1}{2} r+\frac{1}{2}\right)}{\Gamma\left(\frac{1}{2} a-\frac{1}{2} i+\frac{1}{2} r+\frac{1}{2}\right)}, \quad\left(i \in \mathbb{N}_{0}\right) ;
\end{aligned}
$$

- Generalizations of Gauss's second theorem:

$$
\begin{aligned}
& { }_{2} F_{1}\left[\begin{array}{r}
a, b ; \\
\frac{1}{2}(a+b+i+1) ; \\
2
\end{array}\right]=\frac{\Gamma\left(\frac{1}{2}\right) \Gamma\left(\frac{1}{2} a+\frac{1}{2} b+\frac{1}{2} \mathfrak{i}+\frac{1}{2}\right) \Gamma\left(\frac{1}{2} a-\frac{1}{2} b-\frac{1}{2} \mathfrak{i}+\frac{1}{2}\right)}{\Gamma\left(\frac{1}{2} b\right) \Gamma\left(\frac{1}{2} b+\frac{1}{2}\right) \Gamma\left(\frac{1}{2} a-\frac{1}{2} b+\frac{1}{2} \mathfrak{i}+\frac{1}{2}\right)} \\
& \times \sum_{r=0}^{i}\left(\begin{array}{l}
i \\
r
\end{array}\right) \frac{(-1)^{r} \Gamma\left(\frac{1}{2} b+\frac{1}{2} r\right)}{\Gamma\left(\frac{1}{2} a-\frac{1}{2} i+\frac{1}{2} r+\frac{1}{2}\right)}, \quad\left(i \in \mathbb{N}_{0}\right) \text {, }
\end{aligned}
$$

and

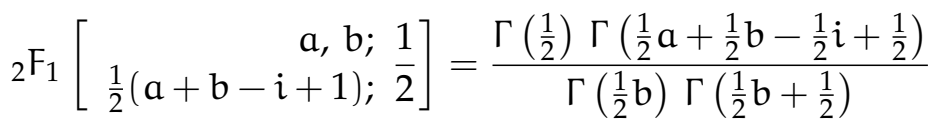

$$
\begin{aligned}
& \times \sum_{r=0}^{i}\left(\begin{array}{l}
i \\
r
\end{array}\right) \frac{\Gamma\left(\frac{1}{2} b+\frac{1}{2} r\right)}{\Gamma\left(\frac{1}{2} a-\frac{1}{2} i+\frac{1}{2} r+\frac{1}{2}\right)}, \quad\left(i \in \mathbb{N}_{0}\right) \text {; }
\end{aligned}
$$

- Generalizations of Bailey's theorem:

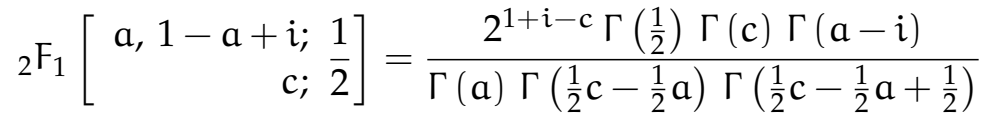

$$
\begin{aligned}
& \times \sum_{r=0}^{i}(-1)^{r}\left(\begin{array}{l}
i \\
r
\end{array}\right) \frac{\Gamma\left(\frac{1}{2} c-\frac{1}{2} a+\frac{1}{2} r\right)}{\Gamma\left(\frac{1}{2} c+\frac{1}{2} a+\frac{1}{2} r-i\right)}, \quad\left(i \in \mathbb{N}_{0}\right) \text {, }
\end{aligned}
$$

and

$$
\begin{aligned}
{ }_{2} F_{1}\left[\begin{array}{r}
a, 1-a-i ; 1 \\
c ; 2
\end{array}\right]= & \frac{2^{1-i-c} \Gamma\left(\frac{1}{2}\right) \Gamma(c)}{\Gamma\left(\frac{1}{2} c-\frac{1}{2} a\right) \Gamma\left(\frac{1}{2} c-\frac{1}{2} a+\frac{1}{2}\right)} \\
& \times \sum_{r=0}^{i}\left(\begin{array}{l}
i \\
r
\end{array}\right) \frac{\Gamma\left(\frac{1}{2} c-\frac{1}{2} a+\frac{1}{2} r\right)}{\Gamma\left(\frac{1}{2} c+\frac{1}{2} a+\frac{1}{2} r\right)}, \quad\left(i \in \mathbb{N}_{0}\right) .
\end{aligned}
$$

The great success of the theory of hypergeometric function of a single variable has inspired the development of a corresponding theory into two and more variables. In 1880, Appell defined four functions known as Appell's functions $F_{1}, F_{2}, F_{3}$ and $F_{4}$. Here we recall $F_{1}$ defined by

$$
F_{1}\left(a, b, b^{\prime} ; c ; x, y\right)=\sum_{m, n=0}^{\infty} \frac{(a)_{m+n}(b)_{m}\left(b^{\prime}\right)_{n}}{(c)_{m+n}} \frac{x^{m}}{m !} \frac{y^{n}}{n !} \quad(\max \{|x|,|y|\}<1),
$$


which is reduced to yield the well-known result:

$$
\begin{gathered}
\mathrm{F}_{1}\left(\mathrm{a}, \mathrm{b}, \mathrm{b}^{\prime} ; \mathrm{c} ; 1,1\right)=\frac{\Gamma(\mathrm{c}) \Gamma\left(\mathrm{c}-\mathrm{a}-\mathrm{b}-\mathrm{b}^{\prime}\right)}{\Gamma(\mathrm{c}-\mathrm{a}) \Gamma\left(\mathrm{c}-\mathrm{b}-\mathrm{b}^{\prime}\right)}, \\
\left(\mathrm{c} \in \mathbb{C} \backslash \mathbb{Z}_{0}^{-} ; \mathfrak{R}\left(\mathrm{c}-\mathrm{a}-\mathrm{b}-\mathrm{b}^{\prime}\right)>0\right) .
\end{gathered}
$$

For a detailed account of the Appell's functions, one may refer to [18, Section 1.6].

Here we recall and repeat a brief history of the birth of Srivastava's triple hypergeometric functions of three variables $\mathrm{H}_{A}, \mathrm{H}_{B}$ and $\mathrm{H}_{C}$ (see [18]). Just as the Gaussian hypergeometric function ${ }_{2} \mathrm{~F}_{1}$ was generalized to ${ }_{p} F_{q}$ by increasing the number of numerator and denominator parameters, the four Appell functions were unified and generalized to the Kampé de Fériet function. Later, Lauricella [6] introduced fourteen complete hypergeometric functions of three variables and of the second order. Lauricella denoted his triple hypergeometric functions by the symbols

$$
\mathrm{F}_{1}, \mathrm{~F}_{2}, \cdots, \mathrm{F}_{14}
$$

of which $F_{1}, F_{2}, F_{5}$ and $F_{9}$ correspond, respectively, to the three-variable Lauricella functions $F_{A}^{(3)}, F_{B}^{(3)}, F_{C}^{(3)}$ and $F_{D}^{(3)}\left(\left[18\right.\right.$, p. 60]). The remaining ten functions $F_{3}, F_{4}, F_{6}, F_{7}, F_{8}, F_{10}, \cdots, F_{14}$ of Lauricella's set apparently fell into oblivion [except that there is an isolated appearance of the triple hypergeometric function $\mathrm{F}_{8}$ in a paper by Mayr [10, p. 265] who came across this function while evaluating certain infinite integrals]. Saran [14] initiated a systematic study of these ten triple hypergeometric functions of Lauricella's set. In the course of a further investigation of Lauricella's fourteen hypergeometric functions of three variables, Srivastava [16] noticed the existence of three additional complete triple hypergeometric functions of the second order. Srivastava denoted his functions by $H_{A}, H_{B}$ and $H_{C}$ which had neither been included in Lauricella's conjecture nor were previously mentioned in the literature. Here, in our present investigation, we recall the series definition of $H_{A}$ as follows (see [18, p. 68]):

$$
\begin{gathered}
H_{A}\left(\alpha, \beta, \beta^{\prime} ; \gamma, \gamma^{\prime} ; x, y, z\right)=\sum_{m, n, p=0}^{\infty} \frac{(\alpha)_{m+p}(\beta)_{m+n}\left(\beta^{\prime}\right)_{n+p}}{(\gamma)_{m}\left(\gamma^{\prime}\right)_{n+p}} \frac{x^{m}}{m !} \frac{y^{n}}{n !} \frac{z^{p}}{p !}, \\
(|x|<r,|y|<s,|z|<t, r+s+t=1+s t) .
\end{gathered}
$$

It is noted that $H_{A}$ provides a generalization of both the Appell's functions $F_{1}$ and $F_{2}$.

Kim et al. [4] obtained the following reducible cases of $\mathrm{H}_{\mathrm{A}}$ by employing the classical summation theorems (1.4), (1.5) and (1.6):

$$
\begin{aligned}
& \mathrm{H}_{\mathrm{A}}\left(\alpha, \beta, \beta^{\prime} ; \gamma, 1+\beta+\beta^{\prime}-\alpha ; 1,1,-1\right) \\
& =\frac{\Gamma(1-\alpha) \Gamma\left(1+\frac{1}{2} \beta^{\prime}\right) \Gamma\left(1+\beta+\beta^{\prime}-\alpha\right) \Gamma(\gamma) \Gamma\left(\gamma-\alpha-\beta+\frac{1}{2} \beta^{\prime}\right)}{\Gamma\left(1+\beta^{\prime}\right) \Gamma(1+\beta-\alpha) \Gamma\left(1+\frac{1}{2} \beta^{\prime}-\alpha\right) \Gamma(\gamma-\beta) \Gamma\left(\gamma-\alpha+\frac{1}{2} \beta^{\prime}\right)} ; \\
& \mathrm{H}_{\mathrm{A}}\left(\alpha, \beta, \beta^{\prime} ; \frac{1}{2}(1+\alpha+\beta)-\frac{1}{4} \beta^{\prime}, 1+\beta+\beta^{\prime}-\alpha ; \frac{1}{2}, 1,-1\right) \\
& =\frac{\Gamma(1-\alpha) \Gamma\left(1+\frac{1}{2} \beta^{\prime}\right) \Gamma\left(1+\beta+\beta^{\prime}-\alpha\right) \Gamma\left(\frac{1}{2}\right) \Gamma\left(\frac{1}{2}+\frac{1}{2} \alpha+\frac{1}{2} \beta-\frac{1}{4} \beta^{\prime}\right)}{\Gamma\left(1+\beta^{\prime}\right) \Gamma(1+\beta-\alpha) \Gamma\left(1+\frac{1}{2} \beta^{\prime}-\alpha\right) \Gamma\left(\frac{1}{2} \beta+\frac{1}{2}\right) \Gamma\left(\frac{1}{2}+\frac{1}{2} \alpha-\frac{1}{4} \beta^{\prime}\right)} ; \\
& \mathrm{H}_{\mathrm{A}}\left(\alpha, \beta, 2 \alpha+2 \beta-2 ; \gamma, \alpha+3 \beta-1 ; \frac{1}{2}, 1,-1\right) \\
& =\frac{\Gamma(1-\alpha) \Gamma(\alpha+\beta) \Gamma(\alpha+3 \beta-1) \Gamma\left(\frac{1}{2} \gamma\right) \Gamma\left(\frac{1}{2} \gamma+\frac{1}{2}\right)}{\Gamma(\beta) \Gamma(1+\beta-\alpha) \Gamma(2 \alpha+2 \beta-1) \Gamma\left(\frac{1}{2} \gamma-\frac{1}{2} \beta\right) \Gamma\left(\frac{1}{2} \gamma-\frac{1}{2} \beta+\frac{1}{2}\right)}
\end{aligned}
$$


Subsequently, Rathie and Kim [13] generalized the results (1.14), (1.15), (1.16) to give explicit expressions of

$$
\begin{gathered}
H_{A}\left(\alpha, \beta, \beta^{\prime} ; 1+\beta-\alpha+\frac{1}{2} \beta^{\prime}+i, 1+\beta+\beta^{\prime}-\alpha ;-1,1,-1\right), \\
H_{A}\left(\alpha, \beta, \beta^{\prime} ; \frac{1}{2}(1+\alpha+\beta+i)-\frac{1}{4} \beta^{\prime}, 1+\beta+\beta^{\prime}-\alpha ; \frac{1}{2}, 1,-1\right),
\end{gathered}
$$

and

$$
H_{A}\left(\alpha, \beta, 2 \alpha+2 \beta-2 i-2 ; \gamma, \alpha+3 \beta-1+2 i ; \frac{1}{2}, 1,-1\right),
$$

( $i=0, \pm 1, \cdots, \pm 5)$ by using generalizations of the classical Kummer's, Gauss second and Bailey's summation theorems which have been derived by Lavoie et al. [7-9].

Here, in this paper, we aim to present generalizations of the results (1.17), (1.18) and (1.19) in the most general form for $i \in \mathbb{N}_{0}$ which are given in six formulas, by making use of generalizations of classical summation theorems (1.7), (1.8), (1.9), (1.10), (1.11), and (1.12). Some known results in [4] and [13] are also pointed out to follow as special cases of our main findings.

\section{Main results}

Here we present six summation formulas for the $\mathrm{H}_{\mathrm{A}}$ in the most general form asserted by the following theorem.

Theorem 2.1. Each of the following six general summation formulas holds true for any $i \in \mathbb{N}_{0}$ :

$$
\begin{aligned}
& H_{A}\left(\alpha, \beta, \beta^{\prime} ; 1+\beta-\alpha+\frac{1}{2} \beta^{\prime}+i, 1+\beta+\beta^{\prime}-\alpha ;-1,1,-1\right) \\
& =\frac{2^{-\beta} \Gamma\left(\frac{1}{2}\right) \Gamma(1-\alpha) \Gamma\left(1+\frac{1}{2} \beta^{\prime}\right) \Gamma\left(1+\beta+\beta^{\prime}-\alpha\right)}{\Gamma\left(1+\beta^{\prime}\right) \Gamma(1+\beta-\alpha) \Gamma\left(1+\frac{1}{2} \beta^{\prime}-\alpha\right) \Gamma\left(\alpha-\frac{1}{2} \beta^{\prime}\right)} \\
& \times \frac{\Gamma\left(\alpha-\frac{1}{2} \beta^{\prime}-i\right) \Gamma\left(1+\beta-\alpha+\frac{1}{2} \beta^{\prime}+i\right)}{\Gamma\left(\frac{1}{2} \beta+\frac{1}{2} \beta^{\prime}-\alpha+\frac{1}{2} i+\frac{1}{2}\right) \Gamma\left(\frac{1}{2} \beta+\frac{1}{2} \beta^{\prime}-\alpha+\frac{1}{2} i+1\right)} \\
& \times \sum_{r=0}^{i}(-1)^{r}\left(\begin{array}{l}
i \\
r
\end{array}\right) \frac{\Gamma\left(\frac{1}{2} \beta+\frac{1}{2} \beta^{\prime}-\alpha+\frac{1}{2} i+\frac{1}{2}+\frac{1}{2} r\right)}{\Gamma\left(\frac{1}{2} \beta-\frac{1}{2} i+\frac{1}{2} r+\frac{1}{2}\right)} \text {, } \\
& \mathrm{H}_{\mathrm{A}}\left(\alpha, \beta, \beta^{\prime} ; 1+\beta-\alpha+\frac{1}{2} \beta^{\prime}-i, 1+\beta+\beta^{\prime}-\alpha ;-1,1,-1\right) \\
& =\frac{2^{-\beta} \Gamma\left(\frac{1}{2}\right) \Gamma(1-\alpha) \Gamma\left(1+\frac{1}{2} \beta^{\prime}\right) \Gamma\left(1+\beta+\beta^{\prime}-\alpha\right)}{\Gamma\left(1+\beta^{\prime}\right) \Gamma(1+\beta-\alpha) \Gamma\left(1+\frac{1}{2} \beta^{\prime}-\alpha\right)} \\
& \times \frac{\Gamma\left(1+\beta-\alpha+\frac{1}{2} \beta^{\prime}-i\right)}{\Gamma\left(\frac{1}{2} \beta+\frac{1}{2} \beta^{\prime}-\alpha-\frac{1}{2} i+\frac{1}{2}\right) \Gamma\left(\frac{1}{2} \beta+\frac{1}{2} \beta^{\prime}-\alpha-\frac{1}{2} i+1\right)} \\
& \times \sum_{r=0}^{i}\left(\begin{array}{l}
i \\
r
\end{array}\right) \frac{\Gamma\left(\frac{1}{2} \beta+\frac{1}{2} \beta^{\prime}-\alpha-\frac{1}{2} \mathfrak{i}+\frac{1}{2}+\frac{1}{2} r\right)}{\Gamma\left(\frac{1}{2} \beta-\frac{1}{2} i+\frac{1}{2} r+\frac{1}{2}\right)}, \\
& \mathrm{H}_{\mathrm{A}}\left(\alpha, \beta, \beta^{\prime} ; \frac{1}{2}(1+\alpha+\beta+i)-\frac{1}{4} \beta^{\prime}, 1+\beta+\beta^{\prime}-\alpha ; \frac{1}{2}, 1,-1\right)
\end{aligned}
$$




$$
\begin{aligned}
& =\frac{\Gamma\left(\frac{1}{2}\right) \Gamma(1-\alpha) \Gamma\left(1+\frac{1}{2} \beta^{\prime}\right) \Gamma\left(1+\beta+\beta^{\prime}-\alpha\right)}{\Gamma\left(1+\beta^{\prime}\right) \Gamma(1+\beta-\alpha) \Gamma\left(1+\frac{1}{2} \beta^{\prime}-\alpha\right)} \\
& \times \frac{\Gamma\left(\frac{1}{2}\left(1+\alpha+\beta-\frac{1}{2} \beta^{\prime}+i\right)\right) \Gamma\left(\frac{1}{2} \beta-\frac{1}{2} \alpha+\frac{1}{4} \beta^{\prime}+\frac{1}{2}-\frac{1}{2} \mathfrak{i}\right)}{\Gamma\left(\frac{1}{2} \alpha-\frac{1}{4} \beta^{\prime}\right) \Gamma\left(\frac{1}{2} \alpha-\frac{1}{4} \beta^{\prime}+\frac{1}{2}\right) \Gamma\left(\frac{1}{2} \beta-\frac{1}{2} \alpha+\frac{1}{4} \beta^{\prime}+\frac{1}{2} i+\frac{1}{2}\right)} \\
& \times \sum_{r=0}^{i}(-1)^{r}\left(\begin{array}{l}
i \\
r
\end{array}\right) \frac{\Gamma\left(\frac{1}{2} \alpha-\frac{1}{4} \beta^{\prime}+\frac{1}{2} r\right)}{\Gamma\left(\frac{1}{2} \beta-\frac{1}{2} i+\frac{1}{2} r+\frac{1}{2}\right)} \\
& \mathrm{H}_{\mathrm{A}}\left(\alpha, \beta, \beta^{\prime} ; \frac{1}{2}(1+\alpha+\beta-i)-\frac{1}{4} \beta^{\prime}, 1+\beta+\beta^{\prime}-\alpha ; \frac{1}{2}, 1,-1\right) \\
& =\frac{\Gamma\left(\frac{1}{2}\right) \Gamma(1-\alpha) \Gamma\left(1+\frac{1}{2} \beta^{\prime}\right) \Gamma\left(1+\beta+\beta^{\prime}-\alpha\right) \Gamma\left(\frac{1}{2}\left(1+\alpha+\beta-\frac{1}{2} \beta^{\prime}-i\right)\right)}{\Gamma\left(1+\beta^{\prime}\right) \Gamma(1+\beta-\alpha) \Gamma\left(1+\frac{1}{2} \beta^{\prime}-\alpha\right) \Gamma\left(\frac{1}{2} \alpha-\frac{1}{4} \beta^{\prime}\right) \Gamma\left(\frac{1}{2} \alpha-\frac{1}{4} \beta^{\prime}+\frac{1}{2}\right)} \\
& \times \sum_{r=0}^{i}\left(\begin{array}{l}
i \\
r
\end{array}\right) \frac{\Gamma\left(\frac{1}{2} \alpha-\frac{1}{4} \beta^{\prime}+\frac{1}{2} r\right)}{\Gamma\left(\frac{1}{2} \beta-\frac{1}{2} i+\frac{1}{2} r+\frac{1}{2}\right)} \\
& \mathrm{H}_{\mathrm{A}}\left(\alpha, \beta, 2 \alpha+2 \beta-2 i-2 ; \gamma, \alpha+3 \beta-1-2 i ; \frac{1}{2}, 1,-1\right) \\
& =\frac{2^{1+i-\gamma} \Gamma\left(\frac{1}{2}\right) \Gamma(1-\alpha) \Gamma(\gamma) \Gamma(\alpha+\beta-i) \Gamma(\alpha+3 \beta-1-2 i)}{\Gamma(\beta) \Gamma(1+\beta-\alpha) \Gamma\left(\frac{1}{2} \gamma-\frac{1}{2} \beta\right) \Gamma\left(\frac{1}{2} \gamma-\frac{1}{2} \beta+\frac{1}{2}\right) \Gamma(2 \alpha+2 \beta-1-2 i)} \\
& \times \sum_{r=0}^{i}(-1)^{r}\left(\begin{array}{l}
i \\
r
\end{array}\right) \frac{\Gamma\left(\frac{1}{2} \gamma-\frac{1}{2} \beta+\frac{1}{2} r\right)}{\Gamma\left(\frac{1}{2} \gamma+\frac{1}{2} \beta+\frac{1}{2} r\right)} \\
& \mathrm{H}_{\mathrm{A}}\left(\alpha, \beta, 2 \alpha+2 \beta+2 i-2 ; \gamma, \alpha+3 \beta-1+2 i ; \frac{1}{2}, 1,-1\right) \\
& =\frac{2^{1-i-\gamma} \Gamma\left(\frac{1}{2}\right) \Gamma(1-\alpha) \Gamma(\gamma) \Gamma(\alpha+\beta+i) \Gamma(\alpha+3 \beta-1+2 i)}{\Gamma(\beta+i) \Gamma(1+\beta-\alpha) \Gamma\left(\frac{1}{2} \gamma-\frac{1}{2} \beta\right) \Gamma\left(\frac{1}{2} \gamma-\frac{1}{2} \beta+\frac{1}{2}\right) \Gamma(2 \alpha+2 \beta-1+2 i)} \\
& \times \sum_{r=0}^{i}\left(\begin{array}{l}
i \\
r
\end{array}\right) \frac{\Gamma\left(\frac{1}{2} \gamma-\frac{1}{2} \beta+\frac{1}{2} r\right)}{\Gamma\left(\frac{1}{2} \gamma+\frac{1}{2} \beta+\frac{1}{2} r\right)} \text {. }
\end{aligned}
$$

Proof. We prove only (2.1). A similar argument will establish the other five formulas, whose detailed accounts of proofs are omitted. Let $\mathcal{L}$ be the left-hand side of (2.1). Using the definition of $\mathrm{H}_{\mathrm{A}}$, we have

$$
\mathcal{L}=\sum_{m, n, p=0}^{\infty} \frac{(\alpha)_{m+p}(\beta)_{m+n}\left(\beta^{\prime}\right)_{n+p}(-1)^{m+p}}{\left(1+\beta-\alpha+\frac{1}{2} \beta^{\prime}+i\right)_{m}\left(1+\beta+\beta^{\prime}-\alpha\right)_{n+p} m ! n ! p !} .
$$

Using the identity $(\lambda)_{m+\ell}=(\lambda)_{m}(\lambda+m)_{\ell}$, we get

$$
\mathcal{L}=\sum_{m=0}^{\infty} \frac{(\alpha)_{m}(\beta)_{m}(-1)^{m}}{\left(1+\beta-\alpha+\frac{1}{2} \beta^{\prime}+i\right)_{m} m !}\left(\sum_{n, p=0}^{\infty}(-1)^{p} \frac{\left(\beta^{\prime}\right)_{n+p}(\alpha+m)_{p}(\beta+m)_{n}}{\left(1+\beta+\beta^{\prime}-\alpha\right)_{n+p} n ! p !}\right) .
$$

Using the definition of the Appell's function $F_{1}(1.13)$, we obtain

$$
\mathcal{L}=\sum_{m=0}^{\infty} \frac{(-1)^{m}(\alpha)_{m}(\beta)_{m}}{\left(1+\beta-\alpha+\frac{1}{2} \beta^{\prime}+i\right)_{m} m !} F_{1}\left(\beta^{\prime}, \beta+m, \alpha+m ; 1+\beta+\beta^{\prime}-\alpha ; 1,-1\right),
$$


which, upon applying the following known formula (see [16, p. 104]):

$$
\mathrm{F}_{1}\left(\mathrm{a}, \mathrm{b}, \mathrm{b}^{\prime} ; 1+\mathrm{a}+\mathrm{b}-\mathrm{b}^{\prime} ; 1,-1\right)=\frac{\Gamma\left(1+\mathrm{a}+\mathrm{b}-\mathrm{b}^{\prime}\right) \Gamma\left(1-\mathrm{b}^{\prime}\right) \Gamma\left(1+\frac{1}{2} \mathrm{a}\right)}{\Gamma(1+\mathrm{a}) \Gamma\left(1+\mathrm{b}-\mathrm{b}^{\prime}\right) \Gamma\left(1+\frac{1}{2} \mathrm{a}-\mathrm{b}^{\prime}\right)},
$$

yields

$$
\begin{aligned}
\mathcal{L}= & \frac{\Gamma\left(1+\frac{1}{2} \beta^{\prime}\right) \Gamma\left(1+\beta+\beta^{\prime}-\alpha\right)}{\Gamma\left(1+\beta^{\prime}\right) \Gamma(1+\beta-\alpha)} \\
& \times \sum_{m=0}^{\infty} \frac{(-1)^{m}(\alpha)_{m}(\beta)_{m}}{\left(1+\beta-\alpha+\frac{1}{2} \beta^{\prime}+i\right)_{m} m !} \frac{\Gamma(1-\alpha-m)}{\Gamma\left(1+\frac{1}{2} \beta^{\prime}-\alpha-m\right)} .
\end{aligned}
$$

Using the identity

$$
\Gamma(\alpha-n)=(-1)^{n} \frac{\Gamma(\alpha)}{(1-\alpha)_{n}}, \quad\left(n \in \mathbb{N}_{0} ; \alpha \in \mathbb{C} \backslash \mathbb{Z}\right),
$$

we have

$$
\begin{aligned}
\mathcal{L}= & \frac{\Gamma(1-\alpha) \Gamma\left(1+\frac{1}{2} \beta^{\prime}\right) \Gamma\left(1+\beta+\beta^{\prime}-\alpha\right)}{\Gamma\left(1+\beta^{\prime}\right) \Gamma(1+\beta-\alpha) \Gamma\left(1+\frac{1}{2} \beta^{\prime}-\alpha\right)} \\
& \times \sum_{m=0}^{\infty} \frac{(-1)^{m}(\beta)_{m}\left(\alpha-\frac{1}{2} \beta^{\prime}\right)_{m}}{\left(1+\beta-\alpha+\frac{1}{2} \beta^{\prime}+i\right)_{m} m !} .
\end{aligned}
$$

Expressing the summation into ${ }_{2} \mathrm{~F}_{1}$, we get

$$
\begin{aligned}
\mathcal{L}= & \frac{\Gamma(1-\alpha) \Gamma\left(1+\frac{1}{2} \beta^{\prime}\right) \Gamma\left(1+\beta+\beta^{\prime}-\alpha\right)}{\Gamma\left(1+\beta^{\prime}\right) \Gamma(1+\beta-\alpha) \Gamma\left(1+\frac{1}{2} \beta^{\prime}-\alpha\right)} \\
& \times{ }_{2} F_{1}\left[\begin{array}{r}
\beta, \alpha-\frac{1}{2} \beta ; \\
1+\beta-\alpha+\frac{1}{2} \beta^{\prime}+i ;
\end{array}-1\right] .
\end{aligned}
$$

Finally applying the generalization of Kummer's summation theorem (1.7) to the ${ }_{2} \mathrm{~F}_{1}$, after some simplification, we obtain the identity (2.1).

The other five formulas (2.2), (2.3), (2.4), (2.5), (2.6) can be established by the same method as in the proof of (2.1). The detailed accounts involving the proofs are omitted to be left to one interested reader.

This completes the proof.

\section{Special cases and concluding remark}

First relevant connections of the main results presented here with those involving relatively simpler formulas are pointed out.

- The special case of (2.1) or (2.2) when $i=0$ yields a known formula [4, Eq. (2.4)].

- Setting $i=0$ in (2.3) or (2.4) reduces to a known formula [4, Eq. (2.5)].

- The special case of (2.5) or (2.6) when $i=0$ yields a known formula [4, Eq. (2.6)].

- Setting $i=1,2,3,4,5$ in (2.1) and (2.2), after some simplification, leads to a known result [13, Eq. (3.1)].

- Taking $i=1,2,3,4,5$ in (2.3) and (2.4), after some simplification, yields a known result [13, Eq. (3.2)].

- Setting $i=1,2,3,4,5$ in (2.5) and (2.6), after some simplification, leads to a known result [13, Eq. (3.3)]. 
For other interesting reducibility of Srivastava's triple hypergeometric series $H_{A}$ and $H_{C}$, one may refer to $[4,5,13]$. The six general formulas for reducibility of the Srivastava's triple hypergeometric function $\mathrm{H}_{\mathrm{A}}$ in terms of Gamma functions presented in compact forms may be (potentially) useful in applications such as applied mathematics and engineering.

\section{Acknowledgment}

This work is dedicated to Professor Yeol Je Cho's 65th birthday anniversary.

\section{References}

[1] G. E. Andrews, R. Askey, R. Roy, Special functions, Encyclopedia of Mathematics and its Applications, Cambridge University Press, Cambridge, (1999). 1

[2] W. N. Bailey, Products of generalized hypergeometric series, Proc. London Math. Soc., 2 (1928), 242-254. 1

[3] W. N. Bailey, Generalized hypergeometric series, Cambridge Tracts in Mathematics and Mathematical Physics, Stechert-Hafner, Inc., New York, (1964). 1

[4] Y. S. Kim, A. K. Rathie, J.-S. Choi, Note on Srivastava's triple hypergeometric series $\mathrm{H}_{\mathrm{A}}$ and $\mathrm{H}_{\mathrm{C}}$, Commun. Korean Math. Soc., 18 (2003), 581-586. 1, 1, 3

[5] Y. S. Kim, A. K. Rathie, J.-S. Choi, Summation formulas derived from the Srivastava's triple hypergeometric series $\mathrm{H}_{\mathrm{C}}$, Commun. Korean Math. Soc., 25 (2010), 185-191. 3

[6] G. Lauricella, Sulle funzioni ipergeometriche a piu variabili, (Italian) Rend. Circ. Mat. Palermo, 7 (1893), 111-158. 1

[7] J.-L. Lavoie, F. Grondin, A. K. Rathie, Generalizations of Watson's theorem on the sum of $a_{3} F_{2}$, Indian J. Math., 34 (1992), 23-32. 1, 1

[8] J.-L. Lavoie, F. Grondin, A. K. Rathie, Generalizations of Whipple's theorem on the sum of a ${ }_{3} \mathrm{~F}_{2}$, J. Comput. Appl. Math., 72 (1996), 293-300.

[9] J.-L. Lavoie, F. Grondin, A. K. Rathie, K. Arora, Generalizations of Dixon's theorem on the sum of $a_{3} \mathrm{~F}_{2}$, Math. Comp., 62 (1994), 267-276. 1, 1

[10] K. Mayr, Über bestimmte integrale und hypergeometriche funktionen, (German) Sitzungsberichte Wien, 141 (1932), 227-265. 1

[11] E. D. Rainville, Special functions, Reprint of 1960 first edition, Chelsea Publishing Co., Bronx, N.Y., (1971). 1

[12] M. A. Rakha, A. K. Rathie, Generalizations of classical summation theorems for the series ${ }_{2} \mathrm{~F}_{1}$ and ${ }_{3} \mathrm{~F}_{2}$ with applications, Integral Transforms Spec. Funct., 22 (2011), 823-840. 1

[13] A. K. Rathie, Y. S. Kim, Further results on Srivastava's triple hypergeometric series $\mathrm{H}_{\mathrm{A}}$ and $\mathrm{H}_{\mathrm{C}}$, Indian J. Pure Appl. Math., 35 (2004), 991-1002. 1, 1, 3

[14] S. Saran, Hypergeometric functions of three variables, Ganita, 5 (1954), 71-91. 1

[15] L. J. Slater, Generalized hypergeometric functions, Cambridge University Press, Cambridge, (1966). 1, 1

[16] H. M. Srivastava, Hypergeometric functions of three variables, Ganita, 15 (1964), 97-108. 1, 2

[17] H. M. Srivastava, J.-S. Choi, Zeta and q-Zeta functions and associated series and integrals, Elsevier, Inc., Amsterdam, (2012). 1

[18] H. M. Srivastava, H. L. Manocha, A treatise on generating functions, Ellis Horwood Series: Mathematics and its Applications, Ellis Horwood Ltd., Chichester; Halsted Press [John Wiley \& Sons, Inc.], New York, (1984). 1 